\title{
COMPOSICIÓN QUÍMICA Y ACTIVIDAD FARMACOLÓGICA DEL EXTRACTO ETANÓLICO DE Satureja sericea (GOYAL)
}

\author{
Chemical composition and pharmacological activity of ethanol extract of Satureja sericea (goyal) \\ Pablo E Bonilla Rivera R ${ }^{1}$, JorgeArroyo A², Nancy M Lozano R ${ }^{1}$, Hamilton Beltrán S ${ }^{3}$, Alex Alba G ${ }^{1}$, Jurissan Aguedo A ${ }^{1}$, Lidia Tinco O ${ }^{1}$, Franco Ríos H ${ }^{1}$ \\ ${ }^{1}$ Instituto de Investigación en Ciencias Farmacéuticas y Recursos Naturales "Juan de Dios Guevara” Facultad de Farmacia y \\ Bioquímica. ${ }^{2}$ Instituto de Investigaciones Clínicas y Laboratorio de Farmacología de la Facultad de Medicina. ${ }^{3}$ Museo de Historia \\ Natural "Javier Prado”. Universidad Nacional Mayor de San Marcos.
}

\section{RESUMEN}

Se estudiaron las partes aéreas de Satureja sericea (goyal) de la comunidad de Mallas, provincia de Huari, Región Ancash, usada para afecciones de las vías digestivas debido a sus propiedades carminativas y antiinflamatorias. La muestra se secó bajo sombra, se pulverizó y se obtuvo el extracto por maceración etanólica. Al realizar el ensayo de solubilidad y marcha fotoquímica se observó gran cantidad de compuestos fenólicos, comprobándose la presencia de cuatro flavonoides mediante reacciones de coloración y precipitación, los que fueron aislados por cromatografía en capa fina a escala preparativa, elucidándose sus estructuras mediante espectroscopía UV visible con reactivos de desplazamiento. El efecto citoprotector de la mucosa gástrica fue determinado por administración oral del extracto alcohólico en ratas y el efecto antisecretor gástrico en ratas sometidas a ligazón pilórica. La seguridad en animales fue evaluada

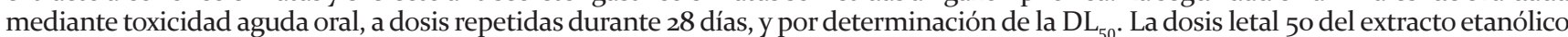
se determinó en ratones, mientras que los parámetros: hematológico, bioquímico e histopatológico, mediante el modelo de toxicidad a dosis repetidas al administrar por vía oral el extracto etanólico en ratas normales según la OECD.

Palabras clave: Satureja sericea (goyal), flavonoides, efecto citoprotector, efecto antisecretor gástrico, toxicidad aguda.

\section{SUMMARY}

We studied the aerial parts of Satureja sericea (goyal) of the Mallas community, Huari province, Ancash Region, used for diseases of the digestive tract due to its carminative and anti-inflammatory properties. The sample was dried under shade, powdered and the extract was obtained by maceration in ethanol. In solubility and phytochemical screening was observed large amount of phenolic compounds. It was confirmed the presence of four flavonoids by color reactions and precipitation; this flavonoids were isolated by thin layer chromatography to preparative-scale and their structures were elucidated by UV visible spectroscopy in ethanol and displacement reagents. The cytoprotective effect on the gastric mucosa was evaluated by oral administration of alcoholic extract in rats. It was also evaluated the gastric antisecretory effect of ethanol extract in rats subjected to pyloric ligation. Safety was evaluated in animals by oral acute toxicity, repeated dose for 28 days abd determining the $\mathrm{DL}_{50}$. The lethal dose 50 of the ethanol extract was determined in mice; the hematological, biochemical and histopathological parameters were evaluated) using the model of repeated dose toxicity when given orally oral ethanol extract to normal rats according to OECD.

Keywords: Satureja sericea (goyal), flavonoids, cytoprotective effect, gastric antisecretory effect, acute toxicity.

\section{INTRODUCCIÓN}

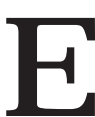
1 Perú esuncampoabiertopara la investigación de su rica flora, evaluada en 17144 especies (gimnospermas y angiospermas), 2458 géneros y 244 familias, distribuidas en las regiones costera, andina y amazónica ${ }^{(1)}$.

Una de estas especies, Satureja sericea "goyal", un arbusto de 50-150 cm de altura, de la familia Lamiaceae, cuyo hábitat es del tipo ladera rocosa ${ }^{(1)}$. Crece en diversas regiones de la sierra del Perú y se usa para aliviar afecciones de las vías digestivas debido a sus propiedades carminativas y antiinflamatorias.)

Actualmenteesnecesariobuscarmetabolitossecundarios o compuestos químicos procedentes de fuentes naturales con actividad antiinflamatoria y de uso en desórdenes digestivos ${ }^{(2,3)}$ como es el caso de los flavonoides, que son compuestos fenólicos distribuidos ampliamente en la naturaleza y han demostrado tener una gran actividad biológica, principalmente como antioxidantes y antiinflamatorios ${ }^{(4,5)}$.

Enesteestudiosepretendedemostrarlaspropiedades de Satureja sericea como citoprotector y antisecretor gástrico. Además se analiza la toxicidad aguda oral, los posibles cambios a nivel hematológico, bioquímico e histopatológico, y los efectos tóxicos ocasionados por la aplicación del modelo de toxicidad a dosis repetida de los componentes presentes en el extracto alcohólico de las partes aéreas de la planta mencionada.

\section{MATERIALES Y MÉTODOS}

\section{Material biológico}

Satureja sericea "goyal": Se clasificaron y seleccionaron las partes aéreas (hojas y ramas) de Satureja sericea "goyal", recolectadas en la comunidad de Mallas, provincia de Huari, al lado oriental de la Cordillera Blanca, en el perímetro del Parque Nacional del Huascarán, departamento de Ancash. Las muestras fueron secadas al ambiente - bajo sombra -, molidas en un molino eléctrico de cuchillas y almacenadas en frascos de vidrio de color ámbar.

Animales de experimentación: Se emplearon 88 Ratas albinas Holtzmann machos de $220 \pm 20$ g; 100 ratones albinos (hembras y machos) de tres meses de edad, peso 
$22 \pm 3 \mathrm{~g}$; 20 ratas albinas Holtzmann (hembras y machos) de tres meses de edad, peso $148 \pm 6 \mathrm{~g}$.

\section{Equipos, instrumentos y reactivos}

Espectrofotómetro UV-visible Hewlett Packard, Equipo de Cromatografía en capa fina, lámpara de luz ultravioleta 265 - 354 1m, estufa Memmert ${ }^{\circledR}$, balanzas analíticas sensibles a $1 \mathrm{~g}$ $\left(\right.$ Hanna $\left.^{\circledR}\right)$; o,1 y o,oooo1 g $\left(\right.$ Bennet $\left.^{\circledR}\right)$, sonda de administración orogástrica, silicagel F254, reactivos y solventes Q.P.

\section{Metodología \\ Análisis fitoquímico}

Se preparó un extracto por maceración de $500 \mathrm{~g}$ de polvo seco de hojas y ramas de Satureja sericea (goyal) en cuatro litros de etanol Q.P. durante ocho días con agitación periódica, el cual se filtró y concentró a sequedad en una estufa de aire circulante a $40{ }^{\circ} \mathrm{C}$. Con el extracto etanólico seco se hizo marcha de solubilidad con solventes de polaridad creciente: éter de petróleo, diclorometano, acetato de etilo, butanol, etanol, metanol y agua destilada; y marcha fitoquímica con reactivos de coloración y precipitación para detectar los principales metabolitos secundarios presentes en el extracto. La identificación de metabolitos secundarios se realizó por cromatografía en capa fina y para el aislamiento se utilizó como fase fija silicagel $\mathrm{F}_{245}$. La estructura química fue elucidada mediante espectrofotometría ultravioleta en etanol con reactivos de desplazamiento: metóxido de sodio, tricloruro de aluminio y ácido clorhídrico; para diferenciar las posiciones de los grupos funcionales en los núcleos químicos presentes ${ }^{(4-7)}$.

Determinación del efecto citoprotector sobre la mucosa gástrica por administración oral del extracto etanólico de Satureja sericea (goyal)

Se utilizaron 48 ratas macho, cepa Holtzmann, de $220 \pm$ $20 \mathrm{~g}$, distribuidos al azar en seis grupos. Previo ayuno de 24 horas, el primer grupo recibió el solvente en dosis de $4 \mathrm{~mL} / \mathrm{Kg}$, el segundo indometacina + alcohol etílico, el tercero omeprazol (10 $\mathrm{mg} / \mathrm{Kg}$ ), los grupos cuarto, quinto y sexto recibieron extracto etanólico de Satureja sericea (goyal) en dosis de 50, 250 y $500 \mathrm{mg} / \mathrm{Kg}$, respectivamente. Los tratamientos con las sustancias de evaluación fueron administrados cada seis horas durante 24 horas. Se indujeron lesiones gástricas una hora después de la primera administración mediante etanol de $96^{\circ}$ (1,o mL/animal, vía oral), y por indometacina (30 mg/Kg, vía subcutánea) ${ }^{(8-12)}$. Los animales fueron sacrificados con pentobarbital $100 \mathrm{mg} /$ $\mathrm{Kg}$, 6 horas después de la administración de indometacina, los estómagos fueron retirados, abiertos por la curvatura mayor y expuestos bajo estereoscopio para registrar el número de lesiones gástricas.

Determinación del efecto antisecrector gástrico por administración intraduodenal del extracto etanólico de Satureja sericea (goyal)

Cuarenta ratas macho, cepa Holtzmann de $220 \pm 20 \mathrm{~g}$, fueron distribuidas al azar en cinco grupos $(n=8)$ y sometidas a ayuno de $24 \mathrm{~h}$ con agua ad libitum. Se les ligó el píloro y se administró el extracto etanólico de Satureja sericea (goyal) a tres grupos en dosis de 50, 250 y $500 \mathrm{mg} / \mathrm{Kg}$, respectivamente; al cuarto grupo se le dio omeprazol $10 \mathrm{mg} / \mathrm{Kg}$ como control positivo y el quinto grupo recibió alcohol $96^{\circ}$ en dosis de 4 $\mathrm{mL} / \mathrm{Kg}$. Por vía intraperitoneal se les administró histamina $50 \mu \mathrm{g} / \mathrm{Kg}$. Cuatro horas más tarde, los animales fueron sacrificados por exposición a éter etílico ${ }^{(13)}$, determinándose el volumen de secreción gástrica $(\mathrm{mL}), \mathrm{pH}$ y cálculo del número de miliequivalentes de iones hidrógeno.

Evaluación de la seguridad en animales, determinación de la dosis letal 50 del extracto etanólico de Satureja sericea (goyal)

Toxicidad aguda oral, determinación de la $\mathrm{DL}_{50}$. Se utilizaron 100 ratones albinos de $22 \pm 3$ g de peso corporal, adquiridos del Instituto Nacional de Salud. Después de una semana de aclimatación, los animales se distribuyeron aleatoriamente en 10 grupos $(\mathrm{n}=10)$, acorde al método ${ }^{(14)}$, administrando el extracto alcohólico en $\mathrm{mg} / \mathrm{Kg}$ de peso corporal en las dosis únicas siguientes: Grupo 2: 500; grupo 3: 10oo; grupo 4: 1500; grupo 5: 2000; grupo 6: 2500; grupo 7: 5000; grupo 8: 60oo; grupo 9: 7000 y grupo 10: 8ooo. Al grupo 1 se le suministraron $10 \mathrm{~mL} / \mathrm{Kg}$ de peso corporal de polisorbato de sodio al 5\%.

Previo ayuno de 12 horas, se administraron los tratamientos por vía oral. Los animales fueron observados individualmente después de la dosificación al menos una vez durante los primeros 30 minutos, periódicamente durante las primeras 24 horas -con especial atención a las primeras ocho-, y de allí en adelante, diariamente por un total de 14 días. Las observaciones de signos tóxicos se registraron sistemáticamente e incluyeron cambios en la piel y pelaje, ojos y membranas mucosas, también en el sistema respiratorio, sistema nervioso autonómico y SNC, actividad somatomotora y patrón de comportamiento. La atención estuvo dirigida a la observación de temblores, convulsiones, salivación, diarrea, letargia, sueño, coma y muerte, a fin de proceder a calcular la $\mathrm{DL}_{50}$ por el método de los Probits ${ }^{(15)}$. Al finalizar este período se procedió a sacrificarlos administrando por vía intraperitoneal pentobarbital en dosis de $100 \mathrm{mg} / \mathrm{Kg}$ de peso.

Determinación de posibles efectos tóxicos del extracto etanólico de Satureja sericea (goyal) a través del modelo de toxicidad a dosis repetidas durante 28 días.

El procedimiento se realizó durante 28 días, según lo estipulado en el ensayo 407 de las directrices de la Organization for Economic Cooperation and Development (OECD) ${ }^{(16)}$, en 20 ratas Holtzmann (10 de cada sexo) de $148 \pm 6$ g, previa aclimatación por 48 horas. El día anterior al ensayo se formaron 2 grupos de 10 animales ( 5 de cada sexo), aplicando el siguiente diseño: Grupo control (I): 4 $\mathrm{mL} / \mathrm{Kg}$ de polisorbato de sodio al 5\%, una vez al día durante 28 días; Grupo tratado (II): extracto etanólico de Satureja sericea (goyal) $100 \mathrm{mg} / \mathrm{Kg}$, una vez al día durante 28 días.

Se observaron diariamente cambios en la piel, pelaje, ojos, y membranas mucosas, así como la presencia de secreciones y excreciones; en la actividad autonómica 
se evidenció lacrimación, piloerección, variaciones en el diámetro de la pupila, patrón respiratorio inusual.

Los animales fueron pesados al inicio y a $\operatorname{los} 7,14,21$ y 28 días. Al finalizar el estudio se promedió el peso de los animales por semana y grupo, teniendo en cuenta el sexo. Las determinaciones hematológicas y de bioquímica clínica se llevaron a cabo a los 28 días de exposición al extracto. La obtención de sangre se realizó por punción cardiaca previo ayuno de 12 horas. Se determinó el número hizo el recuento de hematíes, leucocitos mononucleares y polimorfonucleares; se dosaron hemoglobina, hematocrito, bilirubina total, bilirrubina directa, bilirrubina indirecta, GOT, GPT, fosfatasa alcalina, colesterol total, proteínas totales, albúminas, globulinas, glucosa, úrea y creatinina. Luego fueron sacrificados con sobredosis de pentobarbital sódico (100 mg/Kg).

\section{RESULTADOS}

Se aislaron cuatro componentes con etanol de $96^{\circ}$, y se elucidaron sus estructuras químicas mediante espectroscopía UV-Visible con reactivos de desplazamiento ${ }^{(7)}$ (Tabla 1$)$.

En la evaluación de la toxicidad aguda oral y la determinación de la dosis letal 50 en las dosis ensayadas hasta $8000 \mathrm{mg} / \mathrm{Kg}$ no llegóa producir la muerte de los animales. En la necropsia no se encontraron evidencias macroscópicas de alteraciones en hígado, bazo, riñones, corazón y pulmones. Tampoco hubo diferencias significativas entre los grupos cuando se analizó el peso relativo de los órganos.

Los efectos tóxicos del extracto etanólico de Satureja sericea (goyal) a través del modelo de toxicidad oral a dosis repetidas durante 28 días (OECD), se muestran en las figuras 7-15.

\section{DISCUSIÓN}

Con la marcha de solubilidad se estableció que el extracto alcohólico de Satureja sericea (goyal) contiene componentes solubles en alcoholes y agua, lo cual indica que son de alta polaridad ${ }^{(4,6)}$. Mediante la marcha fitoquímica, encontramos flavonoides, alcaloides y taninos, entre otros ${ }^{(4,6)}$ (Tabla 1). En los ensayos cromatográficos en capa fina analítica se visualizaron varios compuestos fenólicos y, mediante cromatografía en capa fina a escala preparativa, con el sistema de solventes diclorometano:metanol (6:1) se lograron aislar cuatro flavonoides, los que fueron denominados $\mathrm{M}_{1}, \mathrm{M}_{2}, \mathrm{M}_{3}$ y M 4 . Usando espectrofotometría UV-visible en etanol y con reactivos de desplazamiento químico, de acuerdo al método de Mabry et al. ${ }^{(7)}$, se determinó que los falvonoides eran: M1. 3,4,5,7-tetrahidroxi-6,8di metoxi flavona; M2. 7-hidroxi-4,5,6-tri
Tabla 1. Marcha fitoquímica del extracto etanólico de Satureja sericea (goyal).

\begin{tabular}{ccc}
\hline Reactivos & Resultados & Presencia de: \\
\hline Gelatina & + & Taninos \\
Ninhidrina & + & Aminoácidos libres \\
Tricloruro férrico & +++ & Compuestos fenólicos \\
Bortranger & + & Quinonas \\
Mayer & + & Alcaloides \\
Drangendorff & + & Alcaloides \\
Molisch & + & Glicósidos \\
Shinoda & +++ & Flavonoides \\
Lieberman & + & Esteroides y/o Terpenoides
\end{tabular}

$(+++)$ abundante, $(+)$ regular

metoxi flavona; M3. 7-hidroxi-4,5,6,8-tetra metoxi flavona; M4. 3,4,5,7-tetrahidroxi-8-metoxi flavona (Tabla 2).

El extracto etanólico de Satureja sericea (goyal) demostró efecto protector sobre la mucosa gástrica frente a la agresión de la indometacina + alcohol al reducir la hiperemia, las lesiones hemorrágicas y el número de úlceras (Tabla 3, Figuras 1- 3 ) con una p<0,00o1. Con la dosis de 250 $\mathrm{mg} / \mathrm{Kg}$ se ha logrado un mejor efecto citoprotector ${ }^{(8-12)}$.

Mediante la determinación del efecto antisecretorio (13), el extracto alcohólico ha evidenciado reducción de la secreción gástrica en las diferentes dosis utilizadas (Tabla 4, Figura 4) llegando hasta un 49,43\% de eficacia reductora en comparación al grupo control $(\mathrm{p}<0,005)$, con ligero incremento del $\mathrm{pH}$ (Figura 5) y con reducción de la concentración de hidrogeniones (Figura 6). En la toxicidad aguda oral no se observaron signos evidentes de toxicidad por administración del extracto alcohólico

Tabla 2. Longitud de onda máximos en los espectros UV-Visible de cuatro compuestos aislados del extracto etanólico de Satureja sericea (goyal)

\begin{tabular}{|c|c|c|c|c|c|}
\hline \multirow[t]{5}{*}{ M1 } & $\lambda_{\text {máx }}^{\text {EtOH }}$ & : 286; $336 \mathrm{~nm}$ & M2 & $\lambda_{\operatorname{máx}}^{\mathrm{EtOH}}$ & : 278; 330 nm \\
\hline & $\lambda_{\text {máx }}^{\text {Metox.Na }}$ & : 278; 374 nm & & $\lambda_{\text {máx }}^{\text {Metox.Na }}$ & : 274; $364 \mathrm{~nm}$ \\
\hline & $\lambda_{\text {máx }}^{\mathrm{EtOH}+\mathrm{AlCl}_{3}}$ & : 282; $390 \mathrm{~nm}$ & & $\lambda_{\text {máx }}^{\mathrm{EtOH}+\mathrm{AlCl}_{3}}$ & : 278; 330 nm \\
\hline & $\lambda_{\text {máx }}^{\mathrm{EtOH}+\mathrm{AlCl}_{3}+\mathrm{HCl}}$ & : 290; $336 \mathrm{~nm}$ & & $\lambda_{\text {máx }}^{\mathrm{EtOH}+\mathrm{AlCl}_{3}+}$ & : 278; $330 \mathrm{~nm}$ \\
\hline & $\lambda_{\text {máx }}^{\text {EtOH - Actato }}$ & : 284; $330 \mathrm{~nm}$ & & $\lambda_{\text {máx }}^{\text {EtOH - Actato }}$ & : 278; $330 \mathrm{~nm}$ \\
\hline \multirow[t]{5}{*}{ M3 } & $\lambda_{\operatorname{máx}}^{\mathrm{EtOH}}$ & : 284; $330 \mathrm{~nm}$ & M4 & $\lambda_{\text {máx }}^{\mathrm{EtOH}}$ & : 270; $346 \mathrm{~nm}$ \\
\hline & $\lambda_{\text {máx }}^{\text {Metox.Na }}$ & $: \begin{array}{l}286 ; 336 ; 364 \\
\mathrm{~nm}\end{array}$ & & $\lambda_{\text {máx }}^{\text {Metox.Na }}$ & : 274; $404 \mathrm{~nm}$ \\
\hline & $\lambda_{\text {máx }}^{\mathrm{EtOH}+\mathrm{AlCl}_{3}}$ & : 284; $330 \mathrm{~nm}$ & & $\lambda_{\text {máx }}^{\mathrm{EtOH}+\mathrm{AlCl}_{3}}$ & : 276; 388 nm \\
\hline & $\lambda_{\text {máx }}^{\mathrm{EtOH}+\mathrm{AlCl}_{3}+\mathrm{HCl}}$ & : 284; $330 \mathrm{~nm}$ & & $\lambda_{\text {máx }}^{\mathrm{EtOH}+\mathrm{AlCl}_{3}+}$ & : 276; $388 \mathrm{~nm}$ \\
\hline & $\lambda_{\text {máx }}^{\text {EtOH - Actato }}$ & : 284; $330 \mathrm{~nm}$ & & $\lambda_{\text {máx }}^{\text {EtOH - Actato }}$ & $: \begin{array}{l}270 \mathrm{~nm} ; \\
\text { descomposición }\end{array}$ \\
\hline
\end{tabular}


Tabla 3. Efecto citoprotector del extracto etanólico de Satureja sericea (goyal) en ratas con inducción de úlcera gástrica.

\begin{tabular}{|c|c|c|c|c|c|c|c|c|}
\hline \multirow{2}{*}{ Variable } & \multirow{2}{*}{ Tratamiento } & \multirow{2}{*}{$\mathbf{n}$} & \multirow{2}{*}{$\begin{array}{c}\text { Valor } \\
\text { medio }\end{array}$} & \multirow{2}{*}{$\begin{array}{c}\text { Error } \\
\text { estándar }\end{array}$} & \multicolumn{2}{|c|}{ Intérvalo confianza $95 \%$} & \multicolumn{2}{|c|}{ Efecto } \\
\hline & & & & & Inferior & Superior & Mínimo & Máximo \\
\hline \multirow[t]{6}{*}{ Hiperemia } & $\mathrm{SSF} 4 \mathrm{~mL} / \mathrm{kg}$ & 8 & 0,375 & 0,18 & $-0,06$ & 0,81 & 0 & 1 \\
\hline & Indometacina + Alcohol (IA) & 8 & 3,000 & 0,00 & 3,00 & 3,00 & 3 & 3 \\
\hline & $\mathrm{IA}+$ Omeprazol $10 \mathrm{mg} / \mathrm{kg}$ & 8 & 0,750 & 0,16 & 0,36 & 1,14 & 0 & 1 \\
\hline & $\mathrm{IA}+$ Extracto $50 \mathrm{mg} / \mathrm{kg}$ & 8 & 2,125 & 0,35 & 1,30 & 2,95 & 0 & 3 \\
\hline & $\mathrm{IA}+$ Extracto $250 \mathrm{mg} / \mathrm{kg}$ & 8 & 1,250 & 0,25 & 0,66 & 1,84 & 0 & 2 \\
\hline & $\mathrm{IA}+$ Extracto $500 \mathrm{mg} / \mathrm{kg}$ & 8 & 2,125 & 0,40 & 1,18 & 3,07 & 0 & 3 \\
\hline \multirow{6}{*}{$\begin{array}{l}\text { Bandas } \\
\text { hemorrágicas }\end{array}$} & $\mathrm{SSF} 4 \mathrm{~mL} / \mathrm{kg}$ & 8 & 0 & 0,00 & 0,00 & 0,00 & 0 & 0 \\
\hline & Indometacina + Alcohol (IA) & 8 & 4,250 & 0,41 & 3,28 & 5,22 & 3 & 6 \\
\hline & $\mathrm{IA}+$ Omeprazol $10 \mathrm{mg} / \mathrm{kg}$ & 8 & 0,375 & 0,18 & $-0,06$ & 0,81 & 0 & 1 \\
\hline & $\mathrm{IA}+$ Extracto $50 \mathrm{mg} / \mathrm{kg}$ & 8 & 3,000 & 0,27 & 2,37 & 3,63 & 2 & 4 \\
\hline & $\mathrm{IA}+$ Extracto $250 \mathrm{mg} / \mathrm{kg}$ & 8 & 1,625 & 0,46 & 0,54 & 2,71 & 0 & 4 \\
\hline & $\mathrm{IA}+$ Extracto $500 \mathrm{mg} / \mathrm{kg}$ & 8 & 3,125 & 0,44 & 2,08 & 4,17 & 2 & 5 \\
\hline \multirow[t]{6}{*}{ Úlceras } & SSF $4 \mathrm{~mL} / \mathrm{kg}$ & 8 & 0,000 & 0,00 & 0,00 & 0,00 & 0 & 0 \\
\hline & Indometacina + Alcohol (IA) & 8 & 12,875 & 1,61 & 9,07 & 1,68 & 6 & 18 \\
\hline & $\mathrm{IA}+$ Omeprazol $10 \mathrm{mg} / \mathrm{kg}$ & 8 & 1,000 & 0,27 & 0,37 & 1,63 & 0 & 2 \\
\hline & $\mathrm{IA}+$ Extracto $50 \mathrm{mg} / \mathrm{kg}$ & 8 & 5,875 & 0,64 & 4,36 & 7,39 & 3 & 9 \\
\hline & $\mathrm{IA}+$ Extracto $250 \mathrm{mg} / \mathrm{kg}$ & 8 & 2,750 & 1,08 & 0,19 & 5,31 & 0 & 7 \\
\hline & $\mathrm{IA}+$ Extracto $500 \mathrm{mg} / \mathrm{kg}$ & 8 & 4,500 & 1,13 & 1,82 & 7,18 & 0 & 9 \\
\hline
\end{tabular}

Tabla 4. Efecto antisecretorio del extracto etanólico de Satureja sericea (goyal) en ratas con inducción de ligazón pilórica.

\begin{tabular}{|c|c|c|c|c|c|c|c|c|}
\hline \multirow{2}{*}{ Variable } & \multirow{2}{*}{ Tratamiento } & \multirow{2}{*}{$\mathbf{n}$} & \multirow{2}{*}{$\begin{array}{c}\text { Valor } \\
\text { medio }\end{array}$} & \multirow{2}{*}{$\begin{array}{c}\text { Error } \\
\text { estándar }\end{array}$} & \multicolumn{2}{|c|}{ Intérvalo confianza 95\% } & \multicolumn{2}{|c|}{ Efecto } \\
\hline & & & & & Inferior & Superior & Mínimo & Máximo \\
\hline \multirow[t]{5}{*}{ Volumen (mL) } & Ligazón pilórica & 8 & 7,69 & 0,53 & 6,44 & 8,93 & 5,00 & 9,00 \\
\hline & Omeprazole $10 \mathrm{mg} / \mathrm{kg}$ & 8 & 1,38 & 0,16 & 1,00 & 1,75 & 1,00 & 2,00 \\
\hline & Extracto $50 \mathrm{mg} / \mathrm{kg}$ & 8 & 4,63 & 1,18 & 1,84 & 7,41 & 1,20 & 8,60 \\
\hline & Extracto $250 \mathrm{mg} / \mathrm{kg}$ & 8 & 4,44 & 0,87 & 2,38 & 6,49 & 0,20 & 8,00 \\
\hline & Extracto $500 \mathrm{mg} / \mathrm{kg}$ & 8 & 3,89 & 1,14 & 1,19 & 6,58 & 1,00 & 9,00 \\
\hline \multirow[t]{5}{*}{$\mathrm{pH}$} & Ligazón pilórica & 8 & 1,88 & 0,18 & 1,44 & 2,31 & 1,50 & 2,50 \\
\hline & Omeprazole $10 \mathrm{mg} / \mathrm{kg}$ & 8 & 6,03 & 0,06 & 5,89 & 6,16 & 5,80 & 6,20 \\
\hline & Extracto $50 \mathrm{mg} / \mathrm{kg}$ & 8 & 2,56 & 0,35 & 1,74 & 3,38 & 1,50 & 4,00 \\
\hline & Extracto $250 \mathrm{mg} / \mathrm{kg}$ & 8 & 2,63 & 0,26 & 2,00 & 3,25 & 2,00 & 4,00 \\
\hline & Extracto $500 \mathrm{mg} / \mathrm{kg}$ & 8 & 2,63 & 0,29 & 1,95 & 3,30 & 1,50 & 3,80 \\
\hline \multirow[t]{5}{*}{ iones $\mathrm{H}(\mathrm{mEq})$} & Ligazón pilórica & 8 & 131,86 & 22,91 & 77,68 & 186,04 & 49,25 & 200,82 \\
\hline & Omeprazole $10 \mathrm{mg} / \mathrm{kg}$ & 8 & 0,33 & 0,02 & 0,27 & 0,38 & 0,25 & 0,41 \\
\hline & Extracto $50 \mathrm{mg} / \mathrm{kg}$ & 8 & 59,14 & 17,78 & 17,09 & 101,19 & 2,20 & 116,39 \\
\hline & Extracto $250 \mathrm{mg} / \mathrm{kg}$ & 8 & 41,43 & 11,91 & 13,27 & 69,60 & 0,37 & 87,97 \\
\hline & Extracto $500 \mathrm{mg} / \mathrm{kg}$ & 8 & 11,94 & 1,39 & 8,65 & 15,23 & 4,98 & 14,78 \\
\hline
\end{tabular}

sin llegarse a producir la muerte de los animales con ninguna de las dosis, ensayadas hasta $8000 \mathrm{mg} / \mathrm{Kg}$. En la necropsia no se encontraron evidencias macroscópicas de alteraciones en ninguno de los órganos estudiados. Tampoco hubieron diferencias significativas entre los grupos cuando se analizó el peso relativo de los órganos. En la evaluación de la toxicidad, la dosis letal media $\left(\mathrm{DL}_{50}\right)$ estimada supera ampliamente los $8000 \mathrm{mg} / \mathrm{Kg}$, que según las normas de la Comunidad Europea ${ }^{(15)}$ para la clasificación de la toxicidad aguda oral, se considera no clasificado (No tóxico).

En la determinación de la toxicidad oral a dosis repetidas, en los 28 días de tratamiento con el extracto alcohólico, no se observaron síntomas tóxicos a las dosis administradas, tanto a nivel físico general como de comportamiento, comparado con el grupo control. Tampoco se presentó mortalidad de los animales, ni disminución del peso corporal, y la tendencia al aumento fue una constante durante el estudio en ambos sexos, tal como se muestra en la Figura 7. Los valores obtenidos en los indicadores hematológicos (Figuras 8 al 15), no mostraron alteraciones importantes; las variaciones encontradas están dentro del rango establecido por el grupo control y por la literatura especializada ${ }^{(17)}$.

\section{CONCLUSIONES}

1. El extracto etanólico de Satureja sericea (goyal) tiene efecto protector de la mucosa gástrica frente a la agresión con 


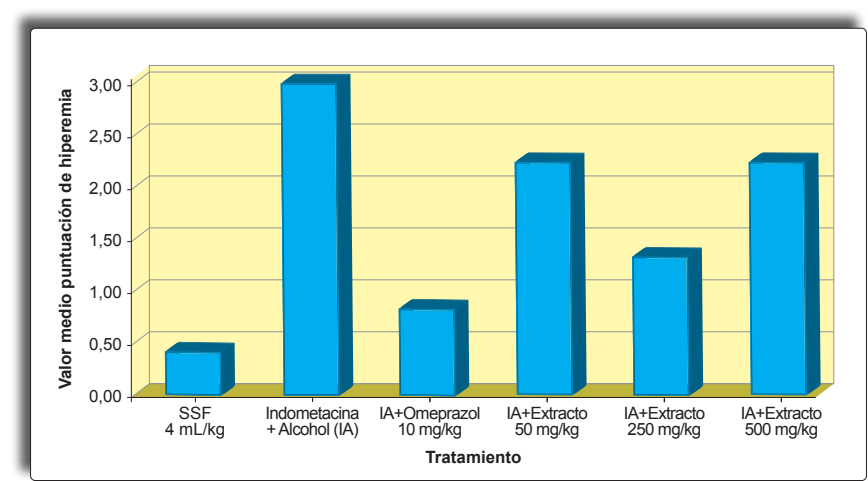

Figura 1. Efecto citoprotector (hiperemia).

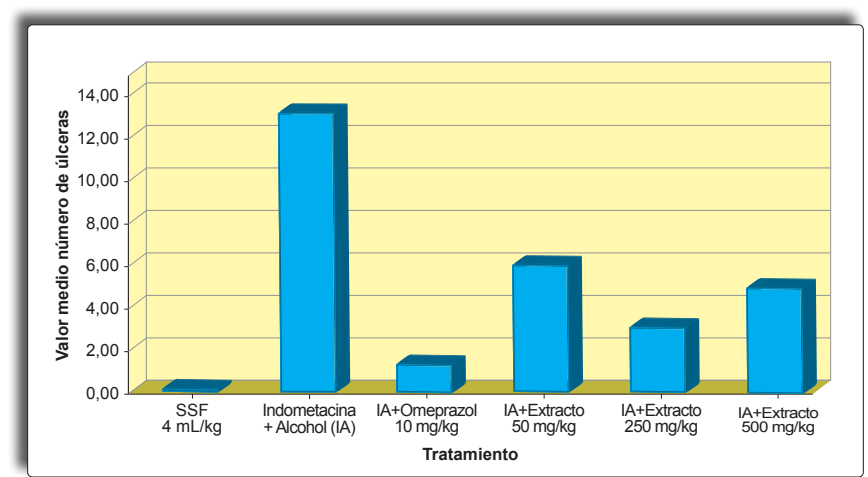

Figura 3. Efecto citoprotector (úlceras).

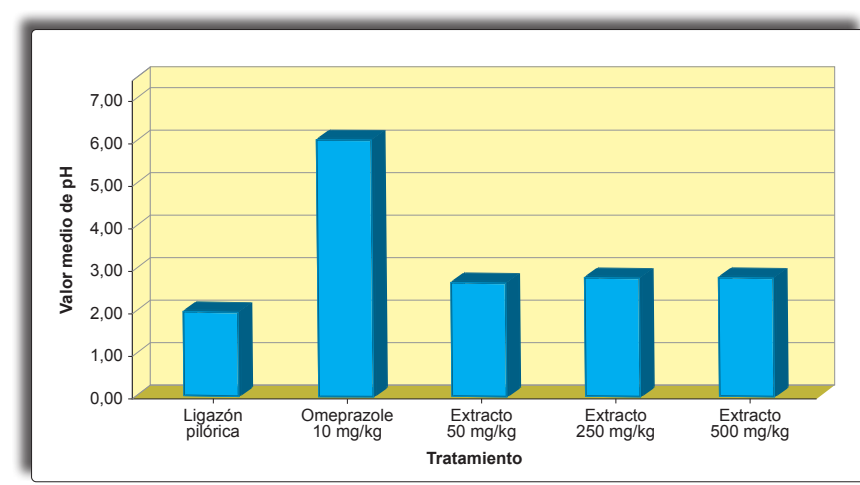

Figura 5. Efecto sobre el pH de la secreción gástrica.

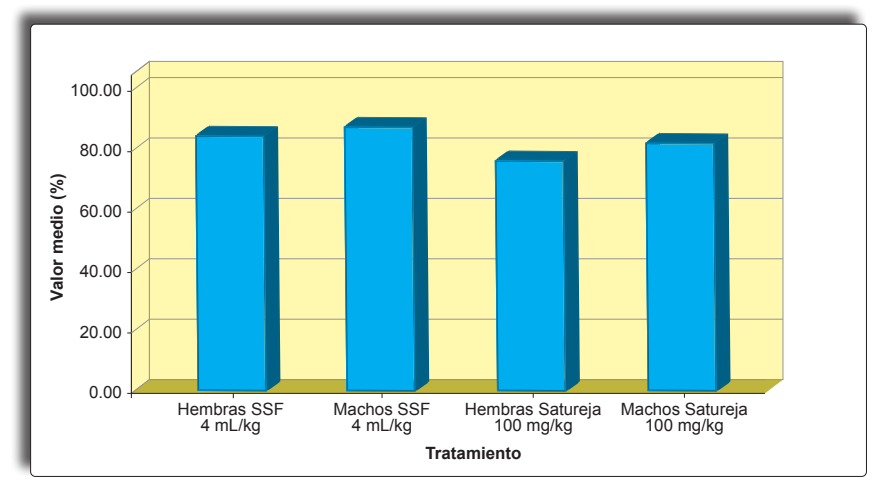

Figura 7. Niveles de mononucleares por administración durante 28 días.

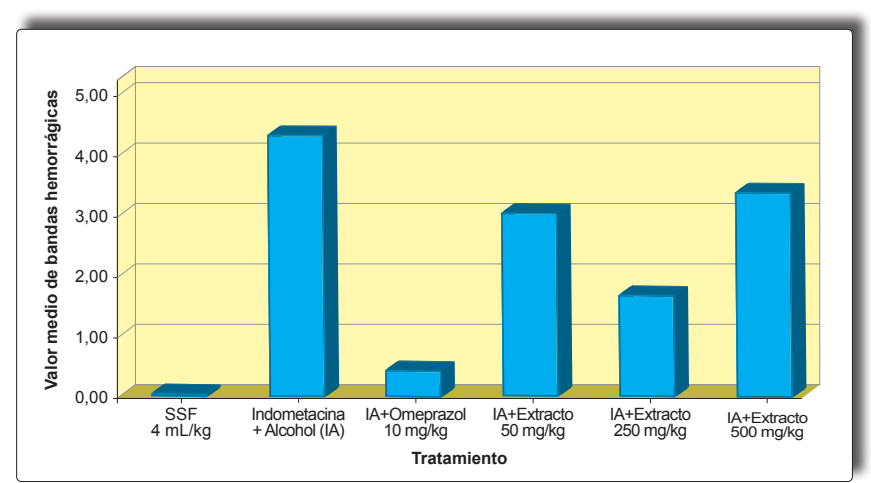

Figura 2. Efecto citoprotector (bandas hemorrágicas).

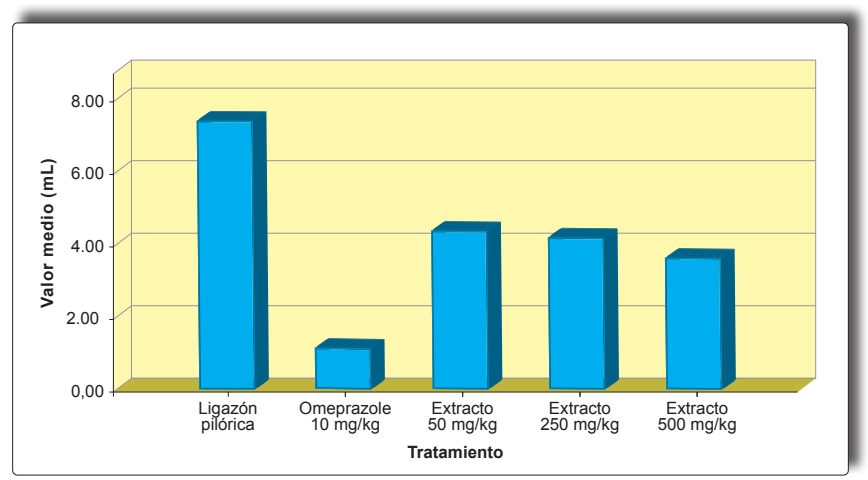

Figura 4. Efecto sobre el volumen de secreción gástrica.

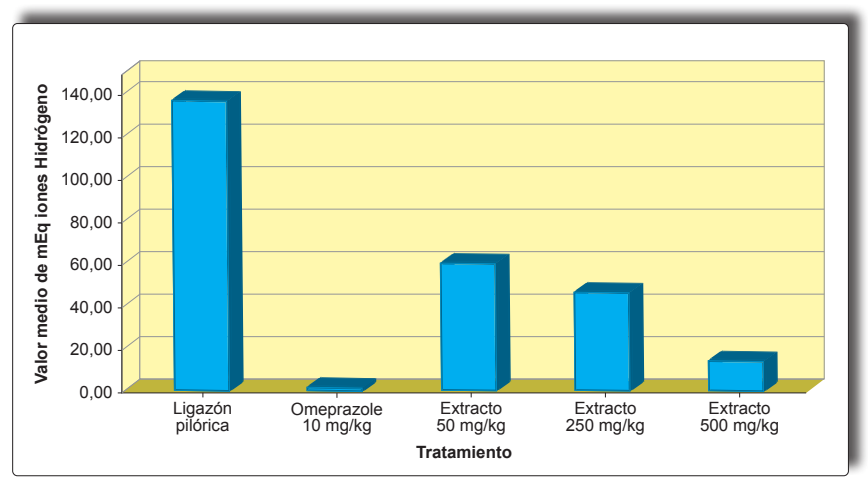

Figura 6. Efecto sobre el nivel de hidrogeniones de la secreción gástrica.

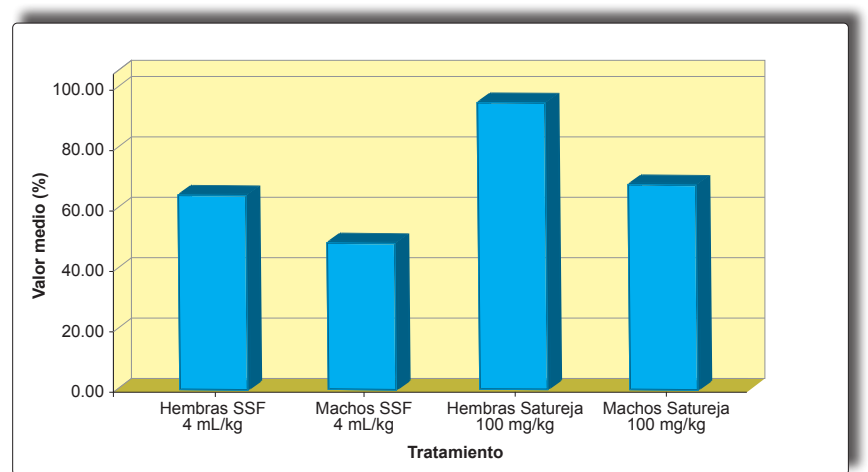

Figura 8. Niveles de polimorfonucleares por administración durante 28 días. 


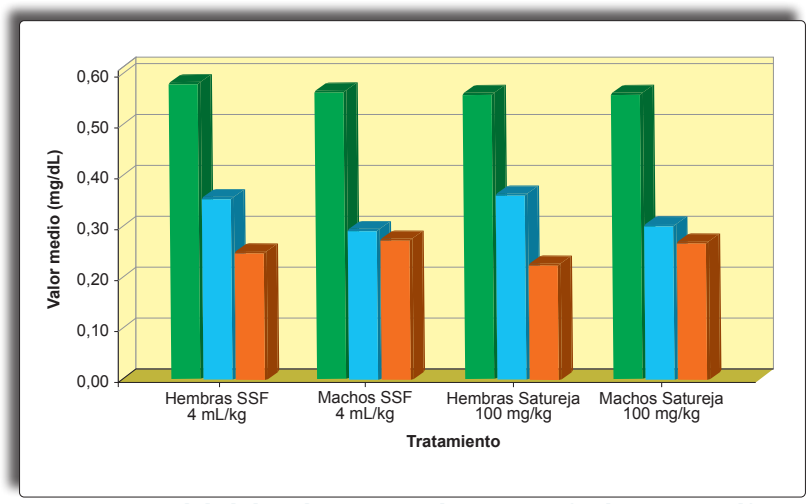

Figura 9. Nivel de bilirrubinas por administración durante 28 días.

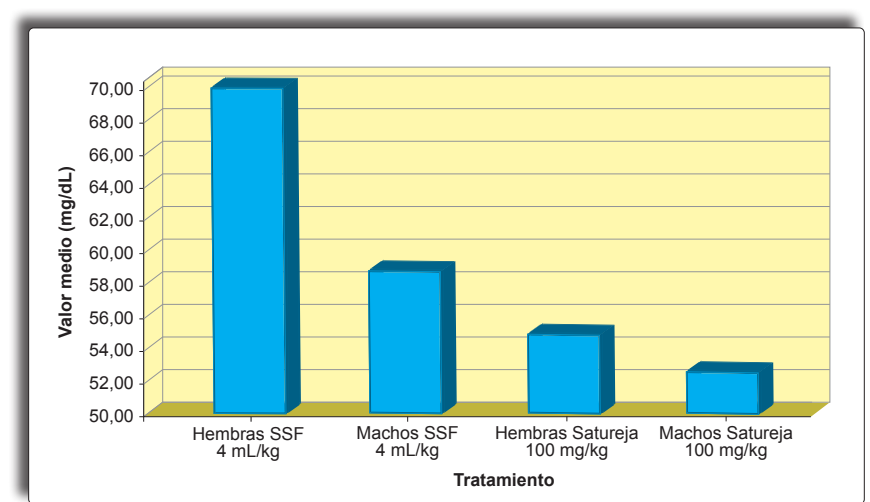

Figura 11. Nivel de colesterol por administración durante 28 días.

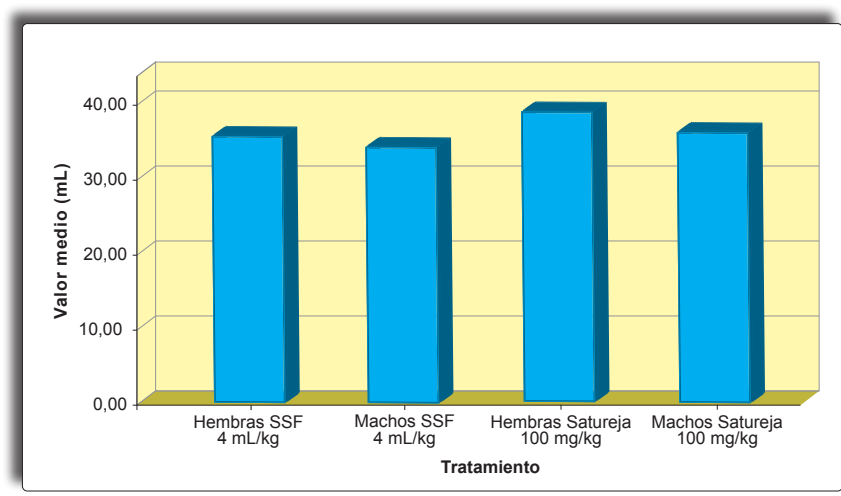

Figura 13. Nivel de úrea por administración durante 28 días.

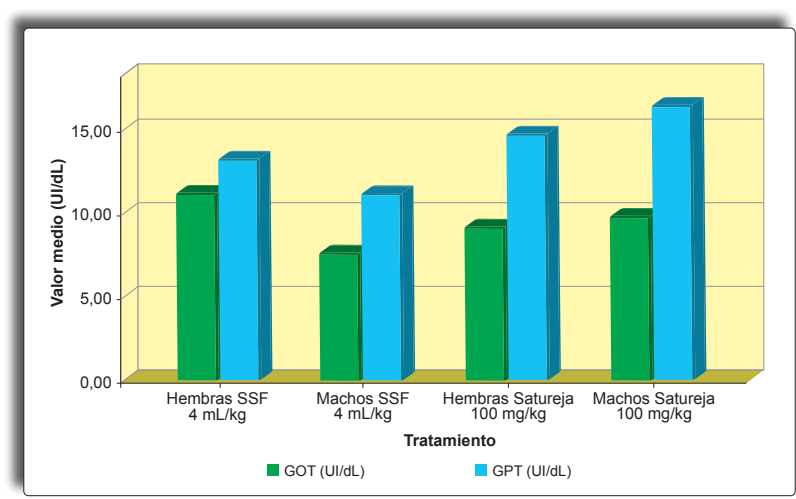

Figura 15. Nivel de transaminasas por administración durante 28 días.

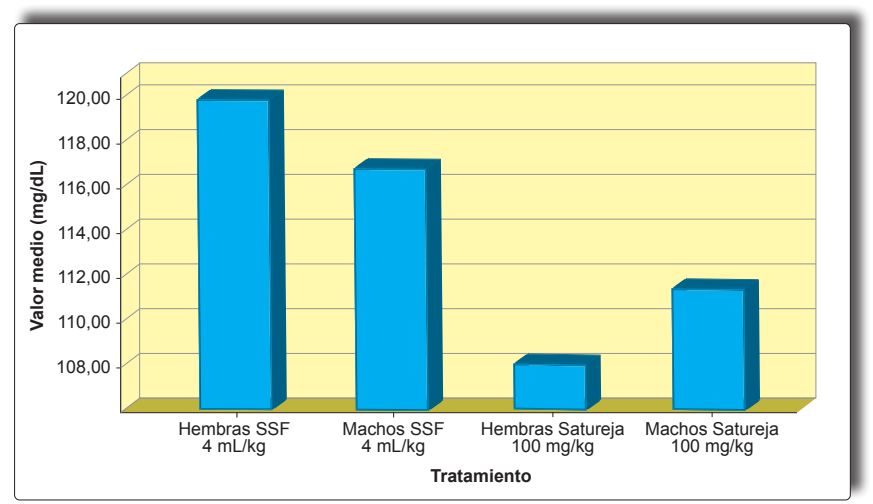

Figura 10. Nivel de bilirrubinas por administración durante 28 días.

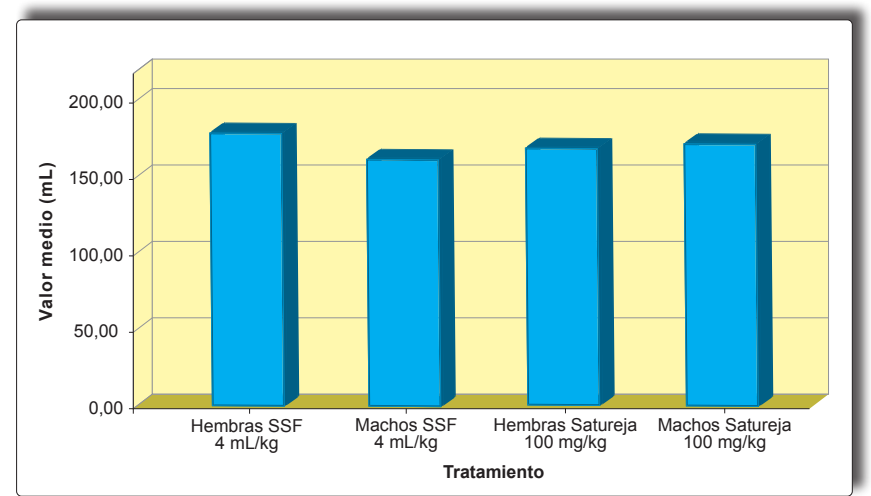

Figura 12. Nivel de fosfatasa alcalina por administración durante 28 días.

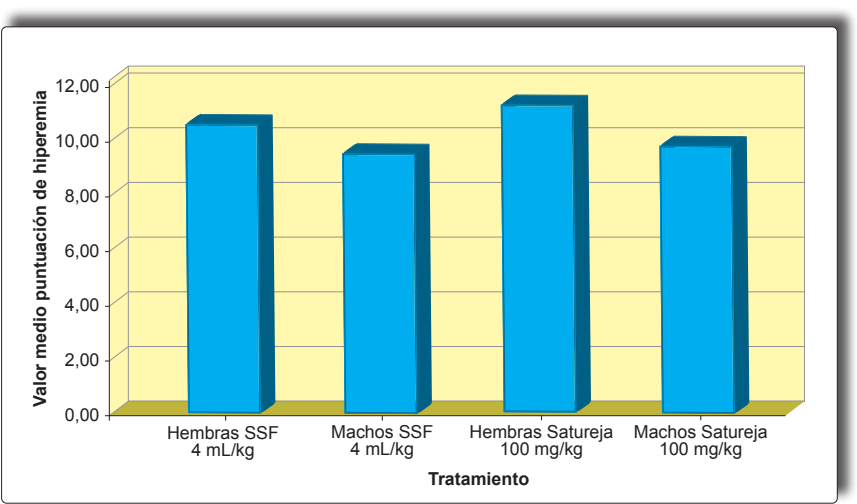

Figura 14. Nivel de creatinina por administración durante 28 días.

indometacina y alcohol, demostrado por la reducción de la hiperemia, las lesiones hemorrágicas y el número de úlceras a dosis de $250 \mathrm{mg} / \mathrm{kg}$, con 78,64\% de efecto citoprotector.

2. El extracto etanólico de Satureja sericea (goyal) ha reducido la secreción gástrica llegando hasta $49,43 \%$ de eficacia reductora comparativamente al grupo control.

3. El estudio de seguridad demostró que el extracto etanólico de las partes aéreas de Satureja sericea (goyal) contiene sustancias no tóxicas, al mostrar en ratones una dosis letal $50\left(\mathrm{DL}_{50}\right)$ por encima de $8000 \mathrm{mg} / \mathrm{Kg}$, además de no producir, a dosis de $100 \mathrm{mg} / \mathrm{Kg}$ durante 28 días, cambios hematológicos ni bioquímicos significativos. 


\section{REFERENCIAS BIBLIOGRÁFICAS}

1. Brako L, Zaruchi JL. Catalogue of Flowering Plants. Angiosperms and Gymnosperms of Perú. Monographs in Sistematic Botany from the Missouri Botanical Garden. Kansas City, 1993.

2. Basten J. Las plantas medicinales de los Kallawayas. ıra. ed. Proyecto Concern Bolivia. La Paz, 1981. p 145.

3. Gupta M. 27o Plantas medicinales iberoamericanas. En: M. Gupta (Ed.), Colombia. Convenio Andres Bello. Bogotá, 1995. p 112, 126, 162.

4. Lock de Ugaz O. Investigación fitoquímica. Métodos en el estudio de productos naturales. 2da ed. Fondo Editorial PUCP. Lima, 1994. p 5.

5. Bruneton J. Plantas medicinales. Fitoquímica y farmacognosia. 2da ed. Acribia S.A. Zaragoza, 2001.

6. Sharapin Nikolai. Fundamentos de tecnología de productos fitoquímicos. Convenio Andrés Bello. Santa Fé de Bogotá, 2000.

7. Mabry TJ, Markham KR, Thomas MB. The systematic identification of flavonoids. Springer-Verlag. New YorkHeidelberg-Berlin, 1970.

8. Djahanguiri B. The production of acute gastric ulceration by indomethacin in the rats. Scandinavian Journal of Gastroenterology 1969; 4: 265.

9. Zhang SR, Cui GJ, Xu RM, Hang C, Guo JY. Antiulcer effect of polycyclicamine compound $\mathrm{HHo1}$ on experimental gastric ulcer in rats. Yao Xue Xue Bao. 1995; 30: 103-6.

10. Liu CF, Lin CC, Lin SC. Protection by tetramethylpyrezine in acute absolute etanol-induced gastric lesions. J Biomed Sci 2002, 9: 395-400.

11. CYTED. Manual de técnicas farmacológicas. Programa Iberoamericano de Ciencia y Tecnología para el Desarrollo. Bogotá, 1999.
12. Arroyo J, Rojas J, Chenguayen J. Manual de modelos experimentales de farmacología. Facultad de Medicina, Universidad Nacional Mayor de San Marcos Lima, 2004.

13. Shay H, Komarov SA, Fels SS, Meranze D, Gruenstein M, Siplet HA. Simple method for the uniform production of gastric ulceration in the rat. Gastroenterology 1945; 5: 43-61.

14. Vega R, Carrillo C. Efecto sobre la motilidad intestinal y toxicidad aguda oral del extracto de Ocimum gratissimum L. (orégano cimarrón). Rev Cubana Plant Med 1997; 2(2-3): 14-18.

15. Unión Europea. Decisión 2000/368/CE de la Comisión: Aproximación de las disposiciones legales, reglamentarias y administrativas en materia de clasificación, embalaje y etiquetado de las sustancias peligrosas. Diario Oficial. Unión Europea. 2000: 108-25.

16. OECD. Guidelines for the testing of chemicals: 407 repeated dose 28-day oral toxicity study in rodents: 28 -day or 14-day study, OECD, París, 2005: 1-7. [Serie en Internet]. [citado 15 May 2008]. Disponible en: http://www.oecd.org

17. Wolford S, Schroer R, Gohs F, Gallo P, Brodeck M, Falk H, et al. Reference range data base for serum chemistry and hematology values in laboratory animals. J Toxicol Environ Health 1986; 18: 161-88.

Manuscrito recibido el: 03/01/2011

Aceptado para su publicación el: 14/04/2011

\section{Correspondencia:}

Nombre: Pablo Enrique Bonilla Rivera

Dirección: Jr. Puno 1002 - Lima o1 - Perú

e-mail: pabloenriquebr@yahoo.com 